\title{
Non-contrast myocardium blood flow: consideration of technical differences between 4D Time-SLIP using tagging aortic root and FAIR
}

\author{
Mitsue Miyazaki ${ }^{*}$, Xiangzhi Zhou', Tsutomu Hoshino' ${ }^{1}$ Kenichi Yokoyama², Rieko Ishimura², Toshiaki Nitatori ${ }^{2}$ \\ From 19th Annual SCMR Scientific Sessions \\ Los Angeles, CA, USA. 27-30 January 2016
}

\section{Background}

A non-contrast 4D Time-Spatial Labeling Inversion Pulse (Time-SLIP) technique [1,2] (3D acquisition and time) has been developed to investigate myocardial bloodflow on healthy volunteers without administration of contrast materials. The technical differences are discussed between our 4D Time-SLIP using tagging aortic root and Flow-sensitive Alternating inversion recovery (FAIR) using a globally IR tagging pulse and control pulse in imaging plane $[3,4]$.

\section{Methods}

The non-contrast 4D Time-SLIP technique was applied on eight healthy volunteers to image myocardium blood flow at $1.5 \mathrm{~T}$. The Time-SLIP sequence has a tagging block with a non-selective inversion recovery (non-selIR) pulse and a spatially selective inversion recovery (sel-IR) and a control block with applying only the nonsel-IR pulse. Both tagging and control were followed by a 3D bSSFP readout. The tagging plane was placed at the proximal ascending aorta and imaging slab was at mid-ventricle. In the tag acquisition, the non-sel-IR pulse inverts the magnetization $(-\mathrm{Mz})$, and the spatially sel-IR pulse immediately inverts back the blood magnetization at the proximal ascending aorta $(+\mathrm{Mz})$. In contrast, the control acquisition applies only the non-sel-IR pulse, which inverts the longitudinal magnetization to $-\mathrm{Mz}$ and experiences exponential $\mathrm{T} 1$ relaxation. The complex subtraction between tag and control depicts only the tagged blood flowing into the myocardium and the background signal was canceled out. Each 3D tag/ control acquisition was performed within a breathhold

${ }^{1}$ MRI, Toshiba Medical Research Institution, USA, Venon Hills, IL, USA

Full list of author information is available at the end of the article and this was repeated for multiple TIs. The time resolved 3D short axis myocardial images were registered and segmented for the visualization of myocardial signal changes caused by the tagged blood along the TI. Perfusion curves were also generated to identify the perfusion peaks.

\section{Results}

Our 4D Time-SLIP results showed that basal to apical blood flow can be observed in all volunteers using the 4D Time-SLIP technique. At the mid-ventricle, the blood flow reaches peak about 200-400 ms after tagging the aortic root blood, and then returned to baseline. This was observed across all volunteers. In comparison, 4D Time-SLIP is a 3D acquisition with time by tagging the blood at aortic root; whereas FAIR is a 2D single slice technique with the blood tagged in a global scale. Furthermore, the myocardial perfusion using FAIR was reported to have a signal tailing effect which did not return to baseline after the peak flow [4]. Our TimeSLIP method showed no tailing signal effect after the peak flow, which may provide useful information for various myocardial perfusion/flow defects.

\section{Conclusions}

Our preliminary results indicate that the 4D Time-SLIP technique permits obtaining non-contrast myocardium perfusion images with high temporal resolution, and may potentially differentiate diseased myocardium from the normal one.

\section{Authors' details}

'MRI, Toshiba Medical Research Institution, USA, Venon Hills, IL, USA.

${ }^{2}$ Radiology, Kyorin University, Tokyo, Japan. 
Published: 27 January 2016

\section{References}

1. Kanazawa H, Miyazaki M: ISMRM 2002.

2. Miyazaki M, et al: Microvasc Res 2015.

3. Zun Z, et al: MRM 2009.

4. Wang JJD, et al: MRM 2010.

doi:10.1186/1532-429X-18-S1-P18

Cite this article as: Miyazaki et al: Non-contrast myocardium blood flow: consideration of technical differences between 4D Time-SLIP using tagging aortic root and FAIR. Journal of Cardiovascular Magnetic Resonance 2016 18(Suppl 1):P18.

Submit your next manuscript to BioMed Central and take full advantage of:

- Convenient online submission

- Thorough peer review

- No space constraints or color figure charges

- Immediate publication on acceptance

- Inclusion in PubMed, CAS, Scopus and Google Scholar

- Research which is freely available for redistribution

Submit your manuscript at www.biomedcentral.com/submit
C Biomed Central 